\title{
Efficacy and safety of febuxostat for treating hyperuricemia in patients with chronic kidney disease and in renal transplant recipients: A systematic review and meta-analysis
}

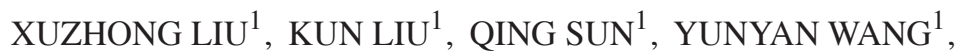 \\ JUNSONG MENG $^{1}$, ZONGYUAN XU ${ }^{1}$ and ZHAOFEI SHI ${ }^{2}$ \\ Departments of ${ }^{1}$ Urology and ${ }^{2}$ Radiology, The Affiliated Huai'an No. 1 People's Hospital \\ of Nanjing Medical University, Huai'an, Nanjing 223300, P.R. China
}

Received February 20, 2018; Accepted April 26, 2018

DOI: $10.3892 /$ etm.2018.6367

\begin{abstract}
Febuxostat is potent and well-tolerated in the management of chronic gout. However, its clinical efficacy and safety in the treatment of hyperuricemia in patients with chronic kidney disease (CKD) and in renal transplant recipients have remained to be fully determined. The MEDLINE, EMBASE and Cochrane Library databases were searched for relevant articles. Data were extracted and pooled results were estimated from the standard mean difference (SMD) with $95 \%$ confidence intervals (95\% CIs). The quality of the studies included was assessed, and their publication bias was examined. Four prospective randomized controlled trials and two retrospective observational studies were included in the systematic review and meta-analysis. Febuxostat administration significantly reduced the serum uric acid concentration in patients with CKD and in renal transplant recipients when compared with allopurinol or placebo in the short-term (1 month: SMD, -2.24 ; 95\% CI, -3.59 to -0.89 ; P-value of $\mathrm{SMD}=0.001 ; I^{2}, 92.4 \%$; 3 months: SMD, $-1.20 ; 95 \%$ CI, -2.04 to -0.36 ; P-value of $\mathrm{SMD}=0.005 ; I^{2}, 88.9 \% ; 6$ months: SMD, $-1.49 ; 95 \% \mathrm{CI},-2.68$ to -0.30 ; P-value of $\mathrm{SMD}=0.014 ; I^{2}$, 92.9\%). Furthermore, the increase in the estimated glomerular filtration rate in the febuxostat group was significantly higher than that in the control group (SMD, 0.30; 95\% CI, 0.031 to
\end{abstract}

Correspondence to: Dr Zhaofei Shi, Department of Radiology, The Affiliated Huai'an No. 1 People's Hospital of Nanjing Medical University, 6 Beijing West Road, Huai'an, Nanjing 223300, P.R. China E-mail: wuhang_nj@163.com

Abbreviations: CKD, chronic kidney disease; UA, uric acid; eGFR, estimated glomerular filtration rate; Scr, serum creatinine; LDL, low-density lipoprotein; HDL, high-density lipoprotein; NOS, Newcastle-Ottawa quality assessment scale; SMD, standard mean difference; $95 \%$ CI, 95\% confidence interval

Key words: febuxostat, hyperuricemia, chronic kidney disease, kidney transplantation, systematic review, meta-analysis
0.58; P-value of $\mathrm{SMD}=0.029 ; I^{2}, 0.0 \%$ ). No significant difference in the changes in serum creatinine ( $\mathrm{Scr}$ ), low-density lipoprotein (LDL) and high-density lipoprotein (HDL) was identified between the two groups (Scr: SMD, -0.17 ; 95\% CI, -0.97 to 0.63 ; P-value of SMD=0.67; $I^{2}, 79.2 \%$; LDL: SMD, $-0.21 ; 95 \% \mathrm{CI},-0.49$ to 0.07 ; P-value of $\mathrm{SMD}=0.13 ; I^{2}$, $34.1 \%$; HDL: SMD, -0.05 ; $95 \%$ CI, -0.70 to 0.61 ; P-value of $\left.\mathrm{SMD}=0.89 ; I^{2}, 69.2 \%\right)$. In conclusion, febuxostat is a potent and well-tolerated agent for the short-term management of hyperuricemia in patients with CKD and in renal transplant recipients. However, these data should be interpreted with caution due to the varied design of the studies included in the present meta-analysis.

\section{Introduction}

Given its high morbidity and mortality rates, chronic kidney disease (CKD) is recognized as a worldwide public health problem (1). Due to the decreased excretion of metabolites, patients with CKD develop various complications, including cardiovascular events, digestive system disorders and endocrine dysfunction $(2,3)$. Of such complications, hyperuricemia, defined as a serum concentration of uric acid (UA) that exceeds the limit of solubility $(7.0 \mathrm{mg} / \mathrm{dl})$, is a common complication that affects $\sim 70 \%$ of hemodialysis patients (4). Several studies on patients with CKD have confirmed the association of hyperuricemia with the development and progression of kidney disease, indicating the requirement for treatment even in the absence of symptoms $(5,6)$.

Febuxostat, a chemically engineered and selective inhibitor of xanthine oxidase, has been approved by the US Food and Drug Administration for the long-term management of hyperuricemia in patients with gout (7). Xanthine oxidase inhibitors, including febuxostat and allopurinol, reduce serum UA levels by impeding the transformation of hypoxanthine to xanthine and of xanthine to UA (8). Structurally differing from allopurinol, which lacks a modified purine ring, febuxostat inhibits the oxidized and reduced forms of xanthine oxidase, whereas oxypurinol, the active metabolite of allopurinol, only inhibits the reduced form of xanthine oxidase (9). Recent evidence-based studies have indicated that febuxostat had a 
better efficacy and safety than other drugs for lowering UA levels, including allopurinol $(10,11)$. However, another study comparing the safety and efficacy of febuxostat and allopurinol in the treatment of chronic gout reported conflicting results (12). These results suggest a lack of consistency in the efficacy and safety of febuxostat and other drugs for treating hyperuricemia.

Experimental and clinical studies have indicated the renoprotective effects of febuxostat against decreased kidney function via inhibition of the formation of renal interstitial fibrosis and macrophage infiltration $(13,14)$. Furthermore, febuxostat is excreted through the urinary and fecal pathways, and may be well-tolerated in the short-term and long-term by patients with CKD with mild to moderate renal dysfunction without a dose reduction, indicating that febuxostat may be superior to other agents used for UA-lowering therapy (15). However, the clinical efficacy and safety of febuxostat in the treatment of hyperuricemia in patients with CKD have remained to be fully determined.

In the present study, a comprehensive, systematic review and meta-analysis was performed to assess the efficacy and safety of febuxostat used for UA-lowering therapy in patients with CKD and renal transplant recipients.

\section{Materials and methods}

Literature search. The present meta-analysis was performed based on the criteria of the Preferred Reporting Items for Systematic Reviews and Meta-Analysis protocol (16). Relevant studies published until April 1, 2017 were identified by a systematic search of the MEDLINE, EMBASE and the Cochrane Library databases, using the following key words: ('chronic kidney disease' OR 'CKD' OR 'end-stage renal disease' OR 'ESRD') OR ('kidney transplantation' OR 'renal transplant') AND ('febuxostat' OR 'TEI 6720' OR 'TEI-6720' OR 'TEI6720') in combination with 'gout' OR 'hyperuricemia'. In addition, the reference lists of eligible studies were scanned to identify potential relevant studies.

Study selection. Original studies evaluating the efficacy and safety of febuxostat in patients with CKD with hyperuricemia were reviewed and selected if they met the following inclusion criteria: i) The study was a randomized or non-randomized controlled trial; ii) patients in the intervention and the control group were diagnosed with CKD or were renal transplant recipients; iii) hyperuricemia was diagnosed unequivocally; and iv) the study included at least one predefined outcome measure. The exclusion criteria were as follows: i) Case reports; ii) reviews; iii) animal experiments, chemical synthesis papers or in vitro studies; iv) studies in a language other than English or Chinese. Two authors (XL and SS) reviewed the articles independently for potential inclusion in the present meta-analysis.

Data extraction. Two authors (XL and SS) extracted the data independently; disputes were resolved by discussion or by consultation of a third investigator (ZX). The following information was extracted from each study: First author, publication year, ethnicity of the subjects, study design, number of cases, age, number of male/female participants, time-points for measurement, inclusion criteria for patients, and treatment in the intervention and control groups. Furthermore, laboratory parameters prior to and after the administration of febuxostat were extracted: UA levels, estimated glomerular filtration rate (eGFR), serum creatinine (Scr), low-density lipoprotein (LDL) and high-density lipoprotein (HDL). The corresponding items in the control group were also extracted.

Quality assessment. The quality of all identified studies was assessed based on the Newcastle-Ottawa quality assessment scale (NOS) (17). The scale included three aspects: Selection of the study population, comparability within the study population and ascertainment of outcomes; the score of each study ranged from 0 to 9 points. The quality of an eligible study was considered high if the total score was $>8$ points and moderate if the total score ranged from 5-7 points.

Statistical analysis. The pooled data were used to assess the efficacy and safety of febuxostat in reducing UA levels in patients with CKD or in renal transplant recipients based on the standard mean difference (SMD) with $95 \%$ confidence intervals (95\% CIs). $\mathrm{P}<0.05$ was considered to indicate a statistically significant difference. Heterogeneity among trials was determined by the $\mathrm{I}^{2}$ test, with $\mathrm{I}^{2}=(\mathrm{Q}-\mathrm{df}) / \mathrm{Q} \times 100 \%$, where $\mathrm{Q}$ was Cochran's heterogeneity statistical parameter and $\mathrm{df}$ the degree of freedom. In cases of low statistical inconsistency $\left(\mathrm{I}^{2}<50 \%\right)$, a fixed-effects model was used for meta-analysis; otherwise, in cases of heterogeneity, a random-effects model was used, which is better adapted to clinical and statistical variations. Sensitivity analysis was performed using the leave-one-out method, which comprises removal of one study at a time and repetition of the analysis. In addition, publication bias was explored using Begg's rank correlation and Egger's weighted regression tests. All statistical analyses were performed using STATA software (release 12.0; StataCorp, College Station, TX, USA).

\section{Results}

Screening and inclusion of studies. In the present meta-analysis, a total of 48 relevant studies were identified, and their titles and abstracts were reviewed. Subsequently, 34 papers were excluded, as they were case reports, reviews or articles written in a language other than English or Chinese. After full-text review of the remaining studies, eight studies were excluded due to study design and the focus of the study being inappropriate (drug cost-effectiveness). Finally, six clinical trials comprising 402 patients were eligible for systematic review and meta-analysis (Fig. 1) (14,18-22), five of which were included for further data analysis $(14,19-22)$. Quantitative statistics were unable to be extracted from the study by Beddhu et al (18) and could only be systematically reviewed.

The clinical characteristics of all included patients included in the present systematic review and meta-analysis are presented in Table I. Among the six studies included, participants in five trials were of Asian ethnicity $(14,19-22)$ and of Caucasian ethnicity in one trial (18). The studies all compared the efficacy of febuxostat with control agents (placebo or allopurinol), and four were randomized 


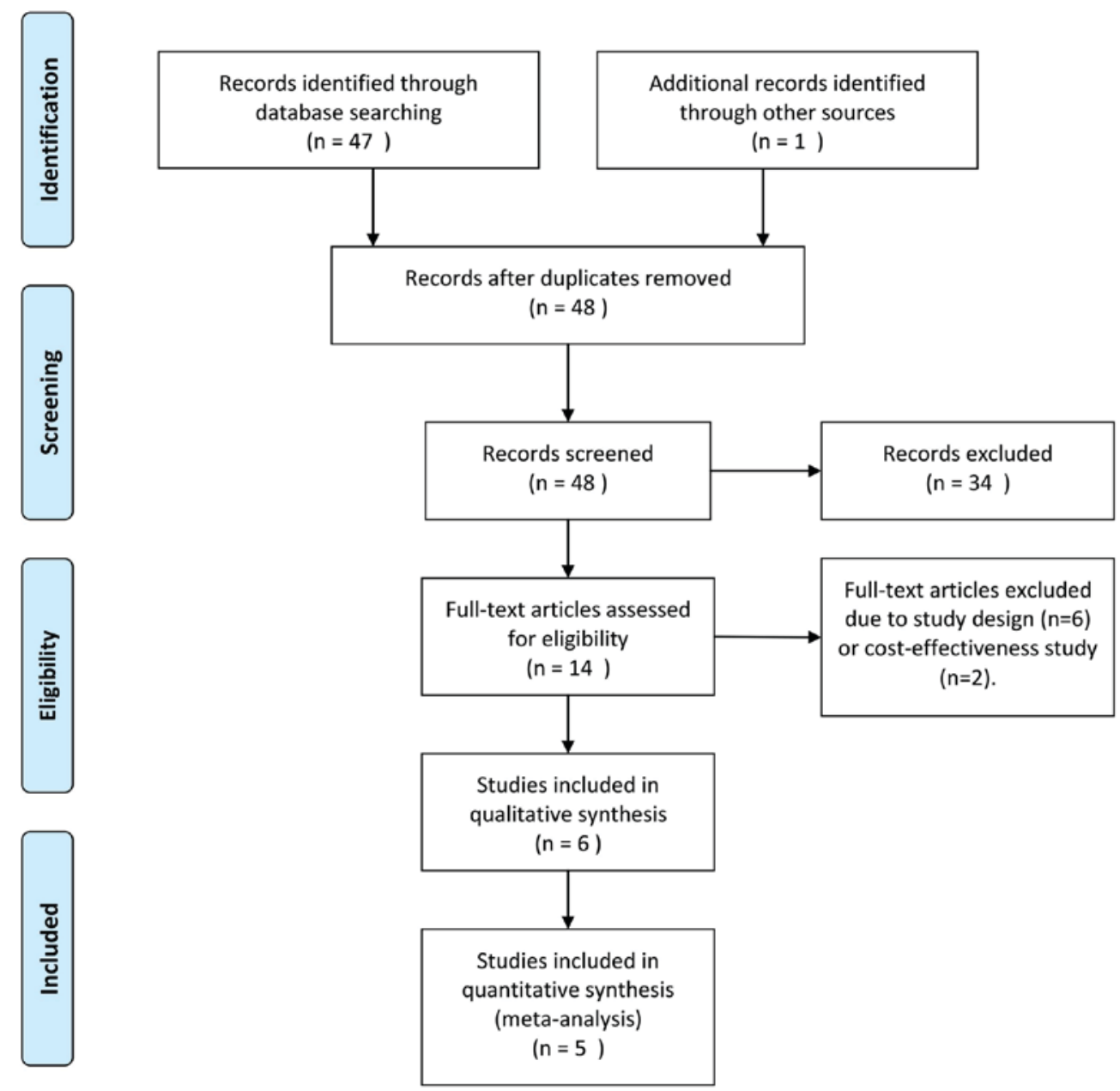

Figure 1. Flow diagram for the selection of studies included in the present systematic review and meta-analysis. Comparisons were made with patients that received allopurinol.

controlled trials $(14,18,20,21)$. More importantly, one trial focused on the effect and safety of febuxostat in renal transplant recipients (19).

Table I also presents the results of the quality assessment of the included studies. Three studies each were deemed as being of high quality and another three as being of moderate quality.

Evaluation of the efficacy of febuxostat in reducing UA. Fig. 2 presents the forest plots for the meta-analysis of UA level reduction in patients with CKD and in renal transplant recipients (The P-values presented in Fig. 2 are representative of heterogeneity). A total of five studies were included in this evaluation (14,19-22). The results of this meta-analysis indicated that febuxostat significantly reduced the UA levels when compared to the control agents (placebo or allopurinol) after 1, 3 and 6 months of administration [1 month: SMD, $-2.24 ; 95 \% \mathrm{CI},-3.59$ to -0.89 ; $\mathrm{P}$-value of $\mathrm{SMD}=0.001 ; \mathrm{I}^{2}$, $92.4 \%$ (Fig. 2A); 3 months: SMD, $-1.20 ; 95 \%$ CI, -2.04 to -0.36; P-value of SMD $=0.005 ; \mathrm{I}^{2}, 88.9 \%$ (Fig. 2B); 6 months: SMD, -1.49 ; $95 \%$ CI, -2.68 to -0.30 ; P-value of $\mathrm{SMD}=0.014$; $\mathrm{I}^{2}, 92.9 \%$ (Fig. 2C)]. However, there was no significant difference in UA levels between the administration of febuxostat and the control agents after 12 months (SMD, -1.58 ; 95\% CI, -3.97 to 0.82 ; P-value of $\mathrm{SMD}=0.196 ; \mathrm{I}^{2}, 96.2 \%$; Fig. 2D).
Evaluation of safety regarding renal function and lipid metabolism. All studies included in the quantitative analysis were compared with allopurinol. The pooled results of four studies $(14,20-22)$ indicated that febuxostat may prevent the kidney function from deteriorating. There was a significantly higher increase in the eGFR in the intervention group compared with that in the control group (SMD, 0.30; 95\% CI, 0.031-0.58; P-value of SMD=0.029; $\mathrm{I}^{2}, 0.0 \%$; Fig. $3 \mathrm{~A}$ ), whereas no significant difference in Scr levels was obtained between the two groups (SMD, -0.17; 95\% CI, -0.97-0.63; P-value of $\mathrm{SMD}=0.67 ; \mathrm{I}^{2}, 79.2 \%$; Fig. 3B). The present study further analyzed the effect of febuxostat on lipid metabolism among the pooled cohort. Compared with the control treatment, the results indicated no significant effect of febuxostat on lipid metabolism, including LDL and HDL [LDL: SMD, -0.21; 95\% CI, -0.49-0.07; P-value of $\mathrm{SMD}=0.13 ; \mathrm{I}^{2}, 34.1 \%$ (Fig. 3C); HDL: SMD, $-0.05 ; 95 \%$ CI, -0.70-0.61; P-value of SMD=0.89; $\mathrm{I}^{2}, 69.2 \%$ (Fig. 3D)].

Sensitivity analysis and publication bias. The results of the leave-one-out sensitivity analyses indicated that none of the studies excluded affected the pooled results, which confirmed that the significance of differences obtained between the two groups was the overall effect of all studies included. Analysis of publication bias revealed that among the studies included 


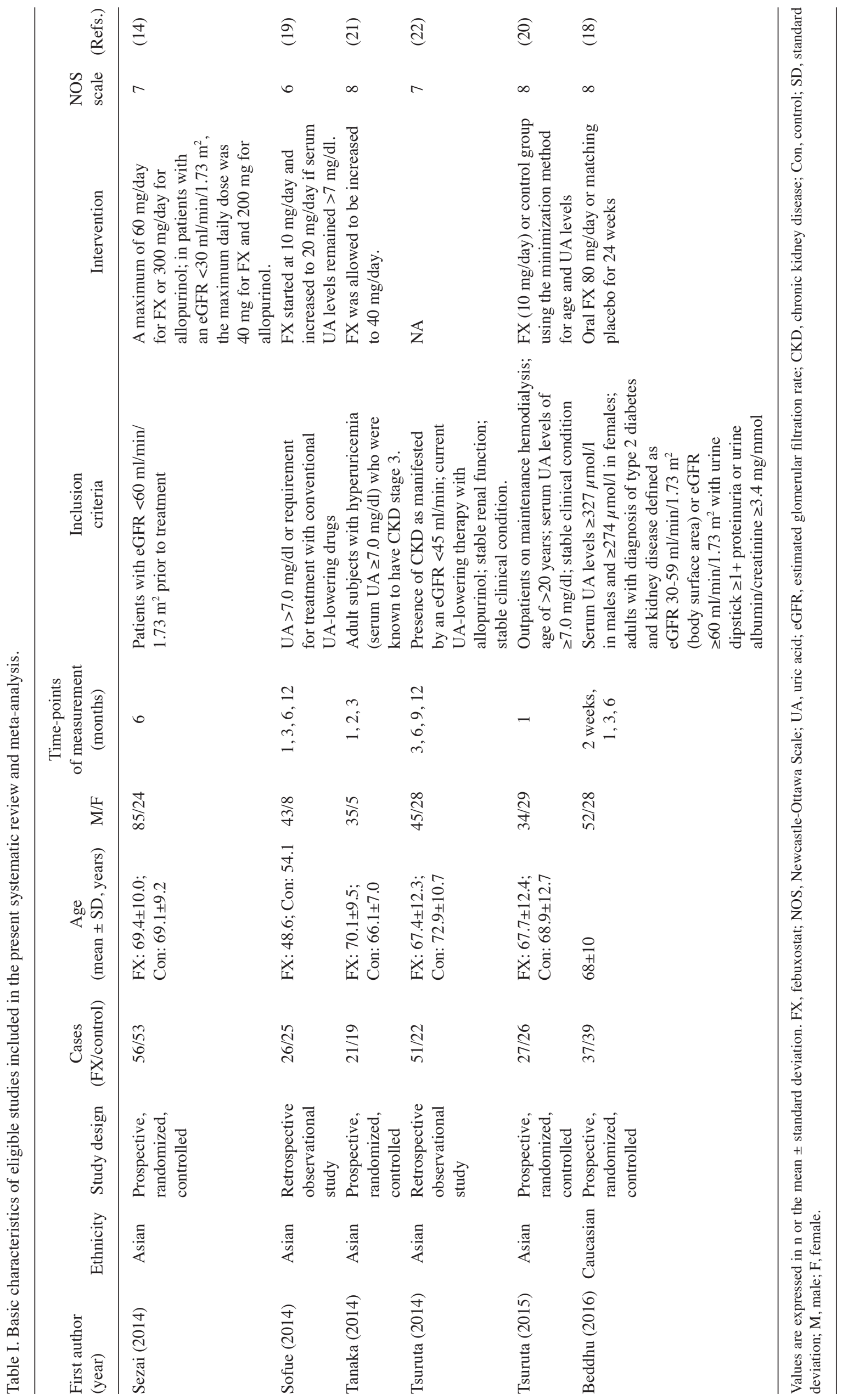



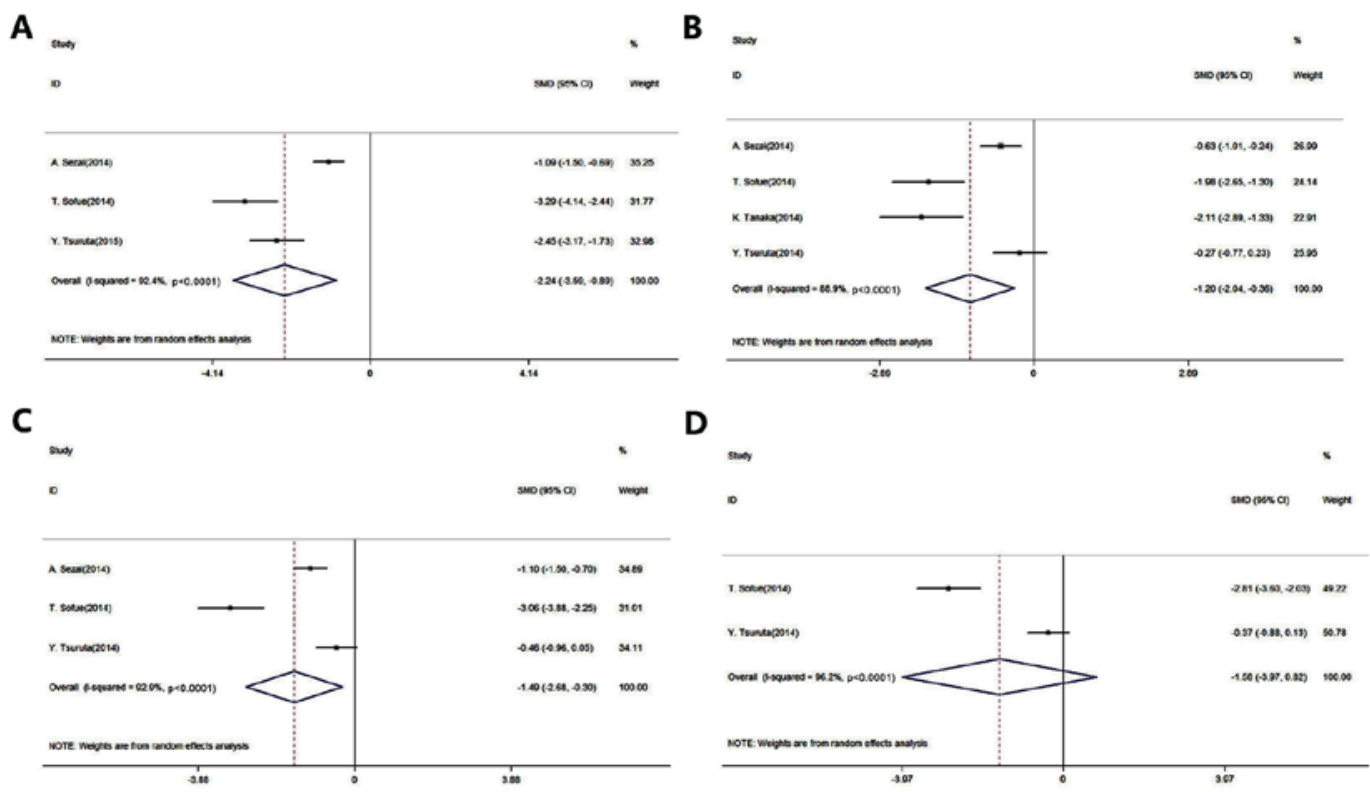

Figure 2. Pooled results of the meta-analysis of the efficacy of febuxostat in treating hyperuricemia compared with allopurinol in patients with chronic kidney disease and in renal transplant recipients after (A) 1, (B) 3, (C) 6 and (D) 12 months of administration. Comparisons were made with patients that received allopurinol. CI, confidence interval; SMD, standard mean difference.
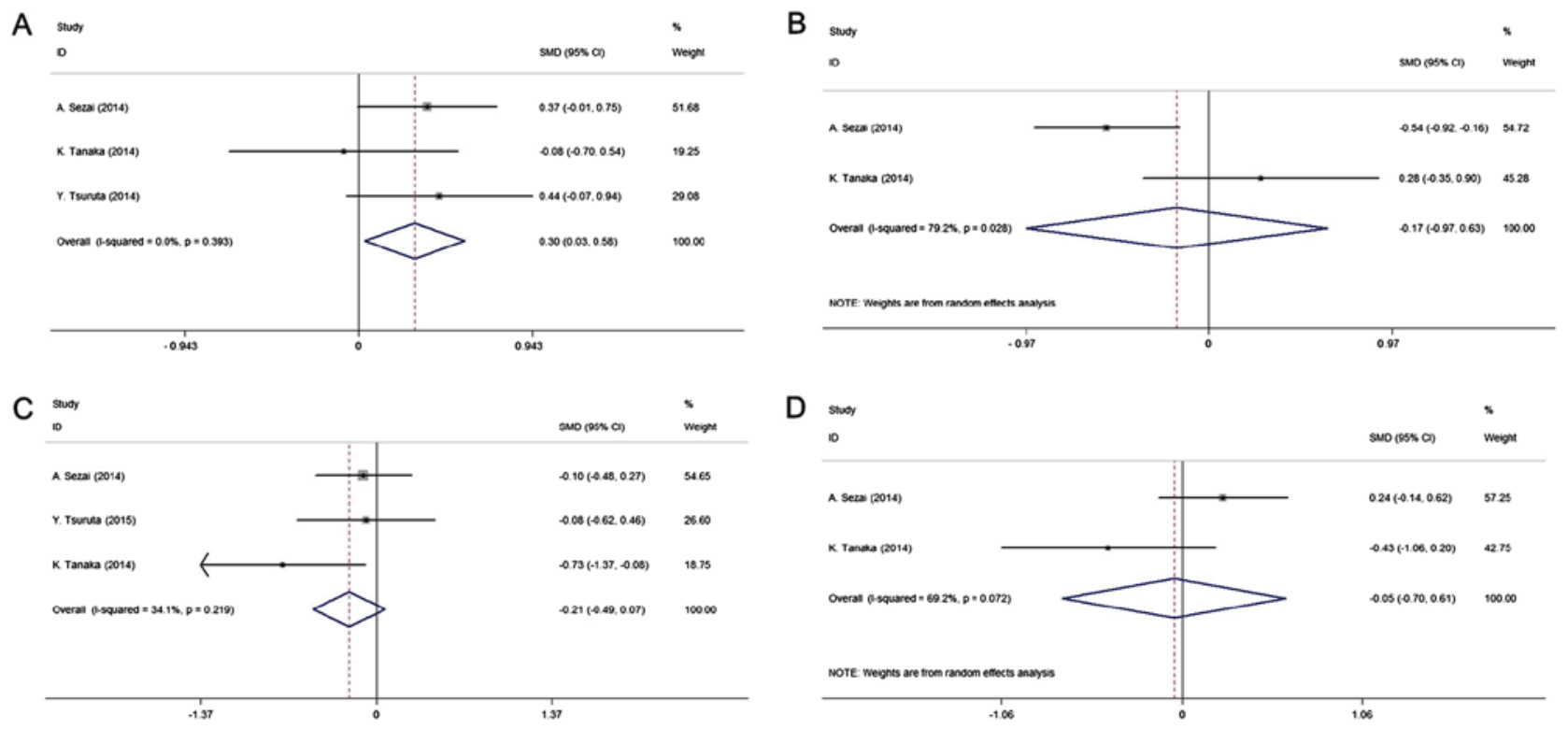

Figure 3. Forest plot of the meta-analysis of the effect of febuxostat on changes in (A) estimated glomerular filtration rate, (B) serum creatinine, (C) low-density lipoprotein and (D) high-density lipoprotein compared with allopurinol (controls). Horizontal lines represent the 95\% confidential CI of each study, the data-points represent the SMD of each study and the diamonds indicate the overall efficacy of FX, including the 95\% CI and SMD. The red dotted lines represent the estimated SMD of FX. CI, confidence interval; SMD, standard mean difference.

in the present meta-analysis, no publication bias was present according to Egger's linear regression and Begg's rank correlation test (Egger's test: $\mathrm{t}=0.18, \mathrm{P}=0.85$; Begg's test: $\mathrm{z}=0.12$, $\mathrm{P}=0.90)$.

\section{Discussion}

Hyperuricemia is a common metabolic complication in patients with CKD and in renal transplant recipients. Studies demonstrating the efficacy and safety of febuxostat in UA-lowering therapy among patients with CKD and in renal transplant recipients have reported conflicting results $(18,23)$. Considering the potential influence of febuxostat on renal function, which has caused the greatest concern of clinicians regarding its use in patients with CKD and kidney transplantation, the data of clinical trials on patients with CKD and renal transplant recipients were combined in the present study. In the present systematic review and meta-analysis, it was revealed that febuxostat significantly reduced serum UA levels and attenuated the decrease in renal function without causing any lipid metabolism-associated disorders in patients with CKD and in renal transplant recipients. 
Febuxostat is an established and effective xanthine oxidative inhibitor alternative to allopurinol, the long-standing gold standard, which remains the most widely prescribed urate-lowering therapy for gout (24). Consistent with the well-established outcomes, the present study indicated that short-term administration (1-6 months) of febuxostat significantly decreased the serum concentrations of UA in patients with CKD and in renal transplant recipients when compared to allopurinol. Furthermore, there was no statistically significant difference in the 12-month administration subgroup, even if the clinical efficacy of febuxostat for reducing UA levels was higher than that of the control group, which received allopurinol. Furthermore, there was significant heterogeneity among the two studies included in the 12-month subgroup analysis, most likely due to the diversity of the included patients, who were patients with CKD and renal transplant recipients, respectively, and due to methodological differences. Consequently, the relatively high heterogeneity may have contributed to the results of the 12-month subgroup analysis. More importantly, the guidelines for the administration of febuxostat for chronic gout recommend dosages of 40-80 mg once daily, with a target serum UA concentration of $<6 \mathrm{mg} / \mathrm{dl}(25,26)$. In the present study, it was observed that the average dosage of febuxostat used in the studies included was $\sim 40 \mathrm{mg} /$ day, and the mean serum level of UA after febuxostat treatment for 12 months was $5.89 \mathrm{mg} / \mathrm{dl}$, indicating that febuxostat administration at $40 \mathrm{mg}$ /day over 12 months may achieve the target serum UA concentration in patients with CKD and in renal transplant recipients. Finally, the present study did not perform any subgroup analysis based on the patients with CKD or renal transplant recipients due to the limited amount of studies. In accordance with our clinical experience, no differences in the treatment regimen were observed between the patients with CKD or renal transplant recipients. However, in patients with CKD, the dosage should be reduced according to their impaired renal function.

Febuxostat is generally well-tolerated and offers the potential advantage of not requiring any dose adjustment in patients with mild to moderate renal impairment $(15,27)$. Consistent with these studies, no obvious difference in the effects of febuxostat and the gold-standard regimen of allopurinol or placebo on the decrease in renal function was observed, which supports the administration of febuxostat to patients with CKD and to renal transplant recipients. The most frequently reported adverse events during these clinical trials were liver dysfunction, hyperlipidemia and gastrointestinal disturbance $(28,29)$. The present meta-analysis study further investigated the effects of febuxostat on lipid metabolism, including the extent of changes in serum LDL and HDL levels. No significant difference was observed between the intervention and control groups, further supporting the administration of the well-established and well-tolerated febuxostat. However, other adverse events, including diarrhea and joint signs and symptoms, still remain to be further investigated.

Of note, the present study has several limitations. The individual studies involved in the systematic review differed in terms of study design, participant ethnicity, inclusion criteria and usage of febuxostat and the administration of the control group (placebo or allopurinol), which significantly contributes to substantial heterogeneity. Doe to the limited number of patients included in the present meta-analysis, it was not possible to further investigate the long-term efficacy of febuxostat on hyperuricemia and certain frequent adverse events in patients with CKD and in renal transplant recipients.

In conclusion, the present meta-analysis study indicated that febuxostat has significant clinical efficacy in reducing serum UA concentrations in the short-term, and has well-tolerated effects on renal function and lipid metabolism in patients with CKD and in renal transplant recipients. These results should be interpreted with caution due to the observational design of the included studies. A well-designed, large-scale, controlled study is required for further clarification.

\section{Acknowledgements}

Not applicable.

\section{Funding}

No funding was received.

\section{Availability of data and materials}

The datasets used and/or analyzed during the current study are available from the corresponding author on reasonable request.

\section{Authors' contributions}

XL contributed to the study design and preparation of the manuscript. KL contributed to the study design and statistical analysis. QS contributed to the statistical analysis and preparation of the manuscript. YW contributed to the statistical analysis. JM contributed to the study design and data collection. $\mathrm{ZX}$ contributed to the statistical analysis and data collection. ZS contributed to the interpretation of data and the preparation of the manuscript. The final version of the manuscript has been read and approved by all authors, and each author believes that the manuscript represents honest work.

\section{Ethical approval and consent to participate}

Not applicable.

\section{Patient consent for publication}

Not applicable.

\section{Competing interests}

The authors declare that they have no competing interests.

\section{References}

1. Woo KT, Choong HL, Wong KS, Tan HB and Chan CM: The contribution of chronic kidney disease to the global burden of major noncommunicable diseases. Kidney Int 81: 1044-1045, 2012.

2. Hasegawa S, Jao TM and Inagi R: Dietary metabolites and chronic kidney disease. Nutrients 9: E358, 2017.

3. Niizuma S, Iwanaga $Y$, Yahata $T$ and Miyazaki $S$ : Renocardiovascular biomarkers: From the perspective of managing chronic kidney disease and cardiovascular disease. Front Cardiovasc Med 4: 10, 2017. 
4. Lee SM, Lee AL, Winters TJ, Tam E, Jaleel M, Stenvinkel P and Johnson RJ: Low serum uric acid level is a risk factor for death in incident hemodialysis patients. Am J Nephrol 29: 79-85, 2009.

5. Weiner DE, Tighiouart H, Elsayed EF, Griffith JL, Salem DN and Levey AS: Uric acid and incident kidney disease in the community. J Am Soc Nephrol 19: 1204-1211, 2008.

6. Li L, Yang C, Zhao Y, Zeng X, Liu F and Fu P: Is hyperuricemia an independent risk factor for new-onset chronic kidney disease? A systematic review and meta-analysis based on observational cohort studies. BMC Nephrol 15: 122, 2014.

7. Takano Y, Hase-Aoki K, Horiuchi H, Zhao L, Kasahara Y Kondo S and Becker MA: Selectivity of febuxostat, a novel non-purine inhibitor of xanthine oxidase/xanthine dehydrogenase. Life Sci 76: 1835-1847, 2005.

8. Chinchilla SP, Urionaguena I and Perez-Ruiz F: Febuxostat for the chronic management of hyperuricemia in patients with gout Expert Rev Clin Pharmacol 9: 665-673, 2016.

9. Frampton JE: Febuxostat: A review of its use in the treatment of hyperuricaemia in patients with gout. Drugs 75: 427-438, 2015

10. Li S, Yang H, Guo Y, Wei F, Yang X, Li D, Li M, Xu W, Li W, Sun L, et al: Comparative efficacy and safety of urate-lowering therapy for the treatment of hyperuricemia: A systematic review and network meta-analysis. Sci Rep 6: 33082, 2016.

11. Sun Y, Li L, Zhou TY and Lu W: A model-based meta-analysis to compare urate-lowering response rate of febuxostat and allopurinol in gout patient. Yao Xue Xue Bao 49: 1674-1683, 2014 (In Chinese).

12. Faruque LI, Ehteshami-Afshar A, Wiebe N, Tjosvold L, Homik J and Tonelli M: A systematic review and meta-analysis on the safety and efficacy of febuxostat versus allopurinol in chronic gout. Semin Arthritis Rheum 43: 367-375, 2013.

13. Omori H, Kawada N, Inoue K, Ueda Y, Yamamoto R, Matsui I, Kaimori J, Takabatake Y, Moriyama T, Isaka Y and Rakugi H: Use of xanthine oxidase inhibitor febuxostat inhibits renal interstitial inflammation and fibrosis in unilateral ureteral obstructive nephropathy. Clin Exp Nephrol 16: 549-556, 2012.

14. Sezai A, Soma M, Nakata K, Osaka S, Ishii Y, Yaoita H, Hata H and Shiono M: Comparison of febuxostat and allopurinol for hyperuricemia in cardiac surgery patients with chronic kidney disease (NU-FLASH trial for CKD). J Cardiol 66: 298-303, 2015.

15. Shekelle PG, Newberry SJ, FitzGerald JD, Motala A, O'Hanlon CE, Tariq A, Okunogbe A, Han D and Shanman R: Management of gout: A systematic review in support of an american college of physicians clinical practice guideline. Ann Intern Med 166: 37-51, 2017.

16. Luís Â, Domingues F and Pereira L: Can cranberries contribute to reduce the incidence of urinary tract infections? A systematic review with meta-analysis and trial sequential analysis of clinical trials. J Urol 198: 614-621, 2017.

17. Stang A: Critical evaluation of the Newcastle-Ottawa scale for the assessment of the quality of nonrandomized studies in meta-analyses. Eur J Epidemiol 25: 603-605, 2010.
18. Beddhu S, Filipowicz R, Wang B, Wei G, Chen X, Roy AC, DuVall SL, Farrukh H, Habib AN, Bjordahl T, et al: A Randomized controlled trial of the effects of Febuxostat therapy on adipokines and markers of kidney fibrosis in asymptomatic hyperuricemic patients with diabetic nephropathy. Can J Kidney Health Dis 3: 2054358116675343, 2016.

19. Sofue T, Inui M, Hara T, Nishijima Y, Moriwaki K, Hayashida Y, Ueda N, Nishiyama A, Kakehi Y and Kohno M: Efficacy and safety of febuxostat in the treatment of hyperuricemia in stable kidney transplant recipients. Drug Des Devel Ther 8: 245-253, 2014.

20. Tsuruta Y, Kikuchi K, Tsuruta Y, Sasaki Y, Moriyama T, Itabashi M, Takei T, Uchida K, Akiba T, Tsuchiya K and Nitta K: Febuxostat improves endothelial function in hemodialysis patients with hyperuricemia: A randomized controlled study. Hemodial Int 19: 514-520, 2015.

21. Tanaka K, Nakayama M, Kanno M, Kimura H, Watanabe K, Tani Y, Hayashi Y, Asahi K, Terawaki H and Watanabe T: Renoprotective effects of febuxostat in hyperuricemic patients with chronic kidney disease: A parallel-group, randomized, controlled trial. Clin Exp Nephrol 19: 1044-1053, 2015.

22. Tsuruta Y, Mochizuki T, Moriyama T, Itabashi M, Takei T, Tsuchiya K and Nitta K: Switching from allopurinol to febuxostat for the treatment of hyperuricemia and renal function in patients with chronic kidney disease. Clin Rheumatol 33: 1643-1648, 2014.

23. Viggiano D, Gigliotti G, Vallone G, Giammarino A, Nigro M and Capasso G: Urate-lowering agents in asymptomatic hyperuricemia: Role of urine sediment analysis and musculoskeletal ultrasound. Kidney Blood Press Res 43: 606-615, 2018.

24. Grassi D, Pontremoli R, Bocale R, Ferri C and Desideri G: Therapeutic approaches to chronic hyperuricemia and gout. High Blood Press Cardiovasc Prev 21: 243-250, 2014.

25. Uloric (febuxostat) tablets (prescribing information). https:// www.ulorichcp.com/. Takeda Pharaceuticals North America, Deerfield, IL, 2009. Accessed June 1st, 2018.

26. Gray CL and Walters-Smith NE: Febuxostat for treatment of chronic gout. Am J Health Syst Pharm 68: 389-398, 2011.

27. Borghi C and Perez-Ruiz F: Urate lowering therapies in the treatment of gout: A systematic review and meta-analysis. Eur Rev Med Pharmacol Sci 20: 983-992, 2016.

28. Becker MA, Schumacher HR Jr, Wortmann RL, MacDonald PA, Eustace D, Palo WA, Streit J and Joseph-Ridge N: Febuxostat compared with allopurinol in patients with hyperuricemia and gout. N Eng J Med 353: 2450-2461, 2005.

29. Schumacher HR Jr, Becker MA, Wortmann RL, Macdonald PA, Hunt B, Streit J, Lademacher C and Joseph-Ridge N: Effects of febuxostat versus allopurinol and placebo in reducing serum urate in subjects with hyperuricemia and gout: A 28-week, phase III, randomized, double-blind, parallel-group trial. Arthritis Rheum 59: 1540-1548, 2008. 